\title{
Mirror mode structures near Venus and Comet P/Halley
}

\author{
D. Schmid ${ }^{1}$, M. Volwerk ${ }^{1}$, F. Plaschke ${ }^{1}$, Z. Vörös ${ }^{1}$, T. L. Zhang ${ }^{1,2}$, W. Baumjohann ${ }^{1}$, and Y. Narita ${ }^{1}$ \\ ${ }^{1}$ Space Research Institute, Austrian Academy of Sciences, 8042 Graz, Austria \\ ${ }^{2}$ University of Science and Technology of China, Hefei, China
}

Correspondence to: D. Schmid (daniel.schmid@oeaw.ac.at)

Received: 28 March 2014 - Revised: 7 May 2014 - Accepted: 8 May 2014 - Published: 17 June 2014

\begin{abstract}
In this paper, we study where mirror mode structures are generated near unmagnetized solar system bodies (Venus and comet P/Halley measured in situ by Venus Express and Giotto). To estimate the location of the mirror mode source region at Venus, we apply a turbulent diffusion model of mirror mode structures, which has already been successfully tested in planetary magnetosheaths (Earth, Jupiter, Saturn). It enables us to estimate the distance between the measured location of the mirror mode and the origin of the mirror mode structure through the mirror mode size. We find that the scenario of mirror mode excitation at the bow shock with subsequent convection and diffusion downstream to the magnetopause is valid for Venus. In the cometary case, however, we find that the size of the mirror mode structure is comparable to the gyroradius of water group ions. This suggests local production of mirror mode structures in the cometary magnetosheath, most likely through fresh ion pickup, as opposed to the convection and diffusion mechanism at Venus.
\end{abstract}

Keywords. Magnetospheric physics (magnetosheath; MHD waves and instabilities)

\section{Introduction}

Mirror modes (MMs) represent non-propagating, compressional structures in a magnetoplasma. They are frequently observed in planetary magnetosheaths of Earth (e.g., Tsurutani et al., 1982; Czaykowska et al., 1998; Baumjohann et al., 1999; Lucek et al., 1999a; Narita et al., 2006), Venus (e.g., Volwerk et al., 2008; Zhang et al., 2009), Jupiter (e.g., Tsurutani et al., 1982; Erdõs and Balogh, 1993; Bavassano-Cattaneo et al., 1998), Saturn (e.g., Tsurutani et al., 1982; Violante et al., 1995), and even near the magnetic pileup boundary of a comet (e.g., Glassmeier et al., 1993).
The MM instability requires high-beta plasma featuring strong temperature anisotropy, i.e., perpendicular temperature higher than parallel temperature (e.g., Hasegawa, 1969; Gary et al., 1993). It should be mentioned that these are also the requirements for the ion cyclotron instability. However, Gary et al. (1993) have shown that the presence of He ions reduces the growth rate of ion cyclotron waves in planetary magnetosheaths, and hence the MM instability will dominate. Recently, it has been found that the presence of electron temperature anisotropy even enhances the mirror mode growth (Remya et al., 2013).

According to Lucek et al. (1999b), temperature anisotropy in the (Earth's) magnetosheath can be created (1) by (multiple) reflection(s) of ions at the bow shock under quasiperpendicular conditions and (2) by compression of the magnetic field in a magnetic pileup region. Both mechanisms increase the gyrotory motion of the ions and the perpendicular temperature with respect to the parallel temperature, resulting in a MM unstable situation. The theoretical scale size of a mirror mode structure in planetary magnetosheaths is $\sim 9 \rho_{\mathrm{p}}$ (proton gyroradii) (Hasegawa and Tsurutani, 2011, and references therein). Yet, in situ spacecraft observations suggest that most of the measured sizes are considerably larger than the theoretical predictions, typical factors ranging between 2 and 10. Hasegawa and Tsurutani (2011) explained this difference by means of a turbulent diffusion process (Bohm-like diffusion): as the MM structures convect away from the region of origin, the large-scale packets undergo turbulent diffusion, while small-scale packets evolve into turbulence subject to selective decay. Hence, the size of MM structures must increase when they are observed downstream of the source region. Based on the equation of diffusion, Hasegawa and Tsurutani (2011) estimated the MM scale size $\lambda$ as a function of the distance $L$ from the source. In their model (henceforth HT-model), the MM size at the source is given by the 
wavelength of maximum growth rate $\lambda_{0} \simeq 9 \rho_{\mathrm{p}}$. The diffusion coefficient is assumed to be of Bohm-type due to strong plasma turbulence. The scale size $\lambda$ of the MM structures at the distance $L$ can then be expressed by

$\lambda(L)=9 \rho_{\mathrm{p}}\left(1+\frac{\omega_{\mathrm{c}, \mathrm{p}} L}{32 u}\right)^{1 / 2}$,

where $u$ denotes the convection speed of the MM structures and $\omega_{\mathrm{c}, \mathrm{p}}$ the proton cyclotron frequency. The HT-model can successfully explain the observed MM sizes in the magnetosheath of magnetized planets (Earth, Jupiter and Saturn) as well as in the heliosphere (Hasegawa and Tsurutani, 2011).

However, for comets, the bow shocks are typically weak and little or no ion heating occurs through the particle's interaction with the shock. Strong perpendicular ion anisotropies rather originate from solar wind pickup of ionized cometary neutrals or field line draping (e.g., Tsurutani et al., 1999, and references therein). We consider only the pickup process, since Delva et al. (2014) recently showed that waves within the magnetic pileup region have properties different from mirror mode waves and field line draping only starts inside the magnetic pileup boundary. One of the dominant ions in the pickup process includes the water group ions $\left(\mathrm{H}_{2} \mathrm{O}^{+}\right)$, where the pickup increases with decreasing distance from the nucleus. Therefore, it can be expected that the generation of the mirror mode structures near comets is more a result from the local pickup process instead of ion-heating at the bow shock and subsequent convection and diffusion.

The purpose of this paper is to determine where MM structures are generated near unmagnetized bodies, in this case Venus and Comet P/Halley, using in situ data from Venus Express and Giotto spacecraft. At Venus we apply the HTmodel to estimate the location of the MM source, by measuring the MM size and calculating the approximate distance to the origin of the MM structure. We want to point out that the goal of this article is not the evaluation of the HT-model for the Venusian magnetosheath. Rather, we take the philosophy that the model is correct and investigate the applicability of the model to our observations. The situation for P/Halley is different. As mentioned above, the dominant ions for MM instability are the (heavy) water group ions. It is found that the observed MM structures at P/Halley are locally generated, as opposed to the convection and diffusion mechanism at Venus.

\section{Mirror mode at Venus and P/Halley}

For the study of MMs at Venus, we use magnetic field data from the fluxgate magnetometer (VEXMAG) at $1 \mathrm{~Hz}$ sampling rate (Zhang et al., 2006) and plasma data from ASPERA (ion mass analyzer) (Barabash et al., 2007) in Venus solar orbital (VSO) coordinates. The ion plasma data have a time resolution of about $3 \mathrm{~min}$, while the MM period is $\sim 3$ to $8 \mathrm{~s}$, as will be shown later. Hence, they cannot be used to identify the MM waves, but they yield the ion plasma temperature $T_{\mathrm{p}}$ and the bulk flow velocity $V_{\mathrm{p}}$ of the plasma that carries the MM structures.

For the study of MMs at P/Halley, the high-resolution Giotto magnetic field data (Neubauer et al., 1986) are used. In order to suppress instrument noise, the data are downsampled from 28 to $1 \mathrm{~Hz}$. Giotto ion data from the ion mass spectrometer (IMS) experiment (Balsiger et al., 1986) and electron data from the Johnstone particle analyzer (JPA) experiment (Johnstone et al., 1987) are taken. They have a resolution of about 8 and $4 \mathrm{~s}$ for ion and electron data, respectively. This is sufficient to resolve the MM structures, as the MM period is $\sim 20$ to $50 \mathrm{~s}$, as will be shown later. But since there are detector field-of-view complications at IMS (see Glassmeier et al., 1993), these data are not reliable for the MM identification. However, to determine the pickup and bulk flow velocity of the dominant $\mathrm{H}_{2} \mathrm{O}^{+}$ions, the pickup speed has been assumed to be the local solar wind velocity of the alpha particles, which is also the bulk flow velocity that carries the MM structure (cf. Glassmeier et al., 1993). Magnetic field and plasma data are given in the Halley-centric solar ecliptic (HSE) coordinate system, where the $X_{\mathrm{HSE}}$ axis points to the $\mathrm{Sun}$ and the $Z_{\mathrm{HSE}}$ axis to the north of the cometary ecliptic plane.

In the absence of high-resolution and/or reliable plasma data, we identify the MM structures following the method of Lucek et al. (1999a, b). Since this method has never been used at comets, we will verify the identified MM with the electron data from JPA. According to this method the MM structures are characterized by

1. large-amplitude fluctuations in the magnetic field magnitude $|\Delta B / B|$. We require $|\Delta B / B| \geq 0.3$.

2. compressible fluctuations, i.e., small angles $\theta_{B \text {,maxvar }}$ between the maximum variance direction of the magnetic disturbance and the background magnetic field $\left(\theta_{B, \text { maxvar }} \leq 30^{\circ}\right.$ Price et al., 1986). We require $\theta_{B, \operatorname{maxvar}} \leq 20^{\circ}$.

3 . wave vectors that are nearly perpendicular to the background magnetic field, i.e., large angles $\beta_{B \text {,minvar be- }}$ tween the minimum variance direction and the background magnetic field. We use $\beta_{B \text {, minvar }} \geq 80^{\circ}$, following Volwerk et al. (2008).

The background field values $B$ are low-pass-filtered magnetic field measurements (Butterworth filter with a cut-off period of $1.5 \mathrm{~min}$ ). The fluctuations $\Delta B$ are given by the difference between measured and low-pass-filtered background magnetic fields. The angles $\theta_{B \text {, maxvar }}$ and $\beta_{B \text {, minvar are ob- }}$ tained from $30 \mathrm{~s}$ long sliding intervals, shifted by $1 \mathrm{~s}$. Minimum variance analysis (MVA; Sonnerup and Scheible, 1998) yields the minimum and maximum variance directions for each window.

Figure 1 shows a sketch of the trajectory of Venus Express and Giotto. The positions of the observed MM structures are 
Table 1. Start and end time of the identified MM with the associated MM period, gyroradius and scale size at Venus and P/Halley.

\begin{tabular}{lclcll}
\hline & MM & Time interval $[\mathrm{UT}]$ & MM period $[\mathrm{s}]$ & Gyroradius $[\mathrm{km}]$ & Measured MM scale size \\
\hline \multirow{2}{*}{ Venus } & A & $06: 40-06: 42$ & 3 & $31 \pm 4$ & $19 \pm 5 \rho_{\mathrm{p}}$ \\
& B & $06: 43-06: 44$ & 8 & $18 \pm 2$ & $28 \pm 7 \rho_{\mathrm{p}}$ \\
\hline \multirow{4}{*}{ P/Halley } & C & $23: 05-23: 07$ & 43 & $2000 \pm 1000$ & $2 \pm 1 \rho_{\mathrm{H}_{2} \mathrm{O}^{+}}$ \\
& D & $23: 13-23: 15$ & 26 & $1600 \pm 800$ & $1 \pm 1 \rho_{\mathrm{H}_{2} \mathrm{O}^{+}}$ \\
& E & $23: 21-23: 23$ & 22 & $1500 \pm 800$ & $1 \pm 1 \rho_{\mathrm{H}_{2} \mathrm{O}^{+}}$ \\
& F & $23: 27-23: 29$ & 43 & $1000 \pm 500$ & $2 \pm 1 \rho_{\mathrm{H}_{2} \mathrm{O}^{+}}$ \\
\hline
\end{tabular}

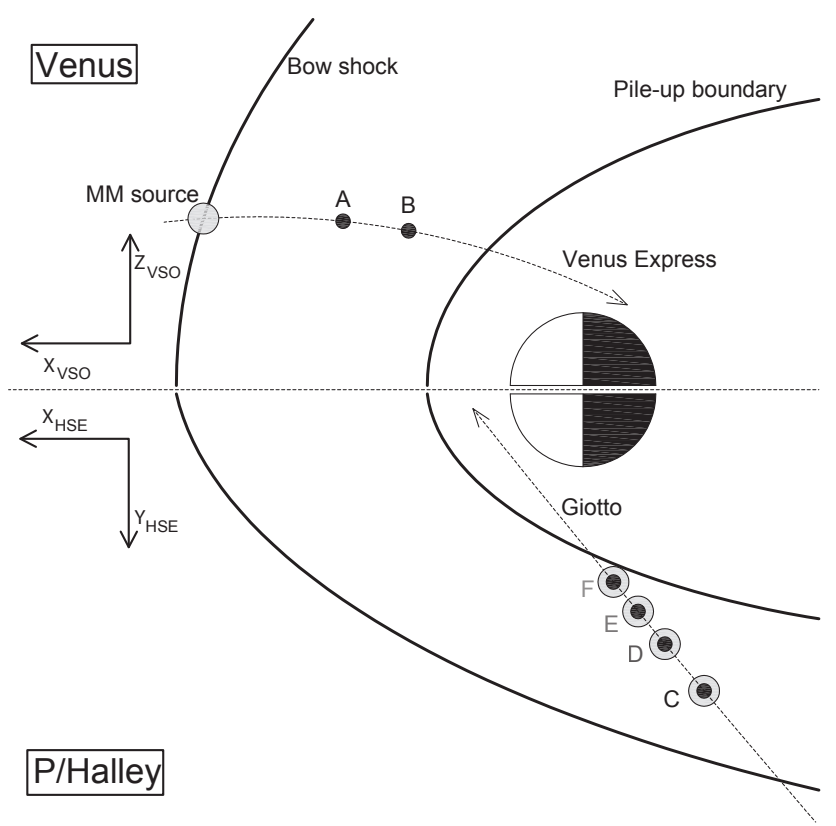

Figure 1. Sketch of the trajectory of Venus Express and Giotto. The position of the observed MM structures are marked by the black dots A-F. The estimated locations of MM generation are marked by the gray shaded circles.

marked by the black dots $\mathrm{A}-\mathrm{F}$, and the estimated locations of MM generation are marked by the gray shaded circles. In Table 1 the start and end time of the identified MM with the associated MM period, gyroradius and scale size at Venus and $\mathrm{P} /$ Halley are given.

\subsection{Venus magnetosheath}

Figure $2 \mathrm{a}$ and $\mathrm{b}$ display the Venus Express magnetic field data and estimated angles $\beta$ and $\theta$ on 28 November 2006 between 06:35 and 06:45 UT. The shaded areas indicate the bow shock crossing (BS) and two identified MM intervals (A and B). Panel a shows the measured magnitude of the magnetic field (in blue) and the low-pass-filtered data (in black).

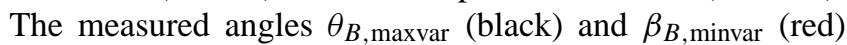
are given in panel b. Figure $2 \mathrm{c}$ shows the total power spectral density $\left(P_{\text {tot }}\right)$ of the magnetic field and in panel $\mathrm{d}$ the ratio between the transverse and total power spectral density $\left(P_{\|} / P_{\text {tot }}\right)$ of the two subintervals (A and $\left.\mathrm{B}\right)$ is given. To obtain the spectra, the magnetic field is transformed into a mean-field-aligned (MFA) coordinate system, and the power spectral density is determined with single-time fast Fourier transform and the error estimate (standard deviation) with the Welch algorithm (see Eriksson, 1998, chapter 1). The vertical dotted lines mark frequencies at which spectral peaks are identified as signatures of the MM structures.

Venus Express entered the magnetosheath at 06:37 UT at the position $(0.98,0.49,0.83)$ in units of planetary radii in VSO coordinates (see Zhang et al., 2007), and approximately followed the flow to where the MMs were observed. MVA of the bow shock crossing yields that the angle between the interplanetary magnetic field (IMF) upstream of the shock and the shock normal is $\sim 107^{\circ}$. This is a quasiperpendicular shock and thus a good precondition for MM instability (e.g., Volwerk et al., 2008). Strong oscillations of the magnetic field are clearly visible after crossing the bow shock. Between 06:40 and 06:42 UT (shaded area A in Fig. 2a, b) the angle $\theta_{B \text {,maxvar }}$ drops well below $20^{\circ}$, indicating that the fluctuations are primarily compressional. At the

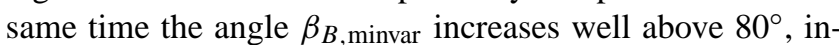
dicating that the wave vectors are quasi-perpendicular to the ambient magnetic field. The spectral analysis yields a clear peak at $0.31 \mathrm{~Hz}$ corresponding to a wave period of about $3 \mathrm{~s}$.

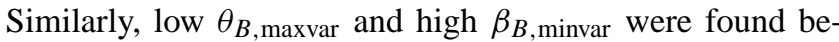
tween 06:43 and 06:44 UT (shaded area B in Fig. 2a, b). The wave period of the MMs of interval $\mathrm{B}$ is about $8 \mathrm{~s}$, frequency at $0.13 \mathrm{~Hz}$.

The flow speed is given by the average proton velocity and is $V_{\mathrm{A}}=(-181,56,-68) \mathrm{km} \mathrm{s}^{-1}$ and $V_{\mathrm{B}}=(-73,24$, $-39) \mathrm{km} \mathrm{s}^{-1}$ for regions $A$ and $B$, respectively. The relative error of the proton velocity and temperature is assumed to be $25 \%$ (Martinecz, 2008). This yields a measured MM size of $19 \pm 5 \rho_{\mathrm{p}}$ and $28 \pm 7 \rho_{\mathrm{p}}$ in $\mathrm{A}$ and $\mathrm{B}$, where the ion gyroradius $\rho_{\mathrm{p}}$ is calculated from the interval median of the magnetic field data and the thermal velocity (gyroradius: $\rho_{\mathrm{p}}=v_{\text {th }} / \omega_{\mathrm{c}, \mathrm{p}}$, where the thermal speed $v_{\text {th }}=\sqrt{2 k_{\mathrm{B}} T / m_{\mathrm{p}}}$ with the Boltzmann constant $k_{\mathrm{B}}$ and proton mass $m_{\mathrm{p}}$ ). Average temperatures of 0.27 and $0.11 \mathrm{MK}$ are used to estimate the thermal velocities. The gyroradii are estimated to be $31 \pm 4 \mathrm{~km}$ and $18 \pm 2 \mathrm{~km}$, respectively. The distances $L$ 


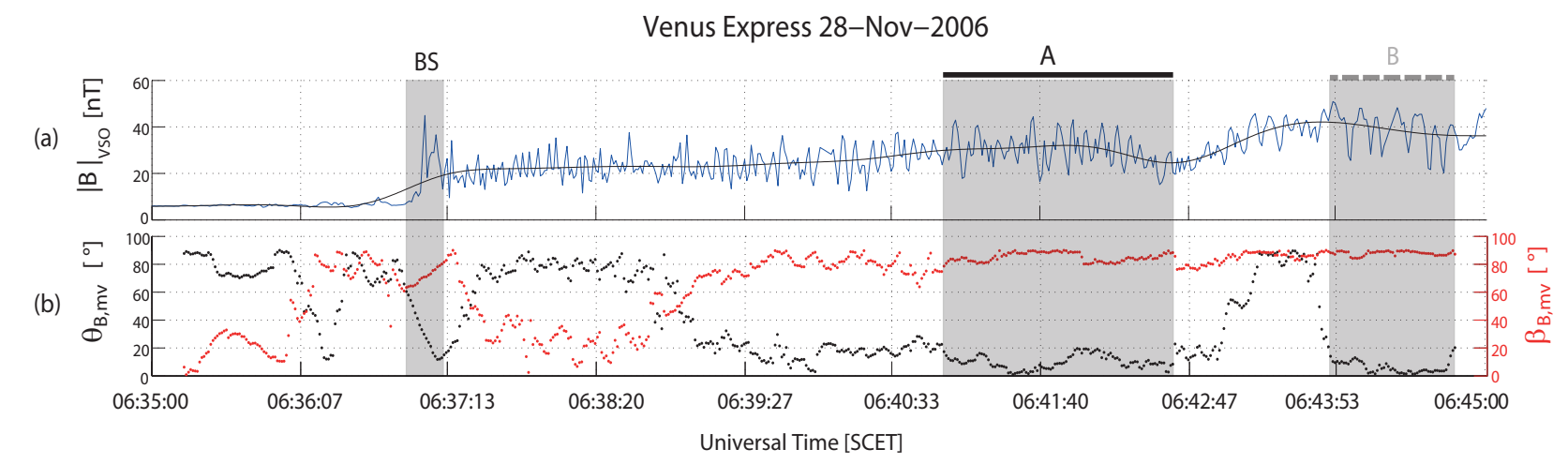

Figure 2. Time series of (a) magnetic field $(|B|)$ and (b) angles between the maximum $(\theta)$ and minimum $(\beta)$ variance directions and the background magnetic field, based on Venus Express data.

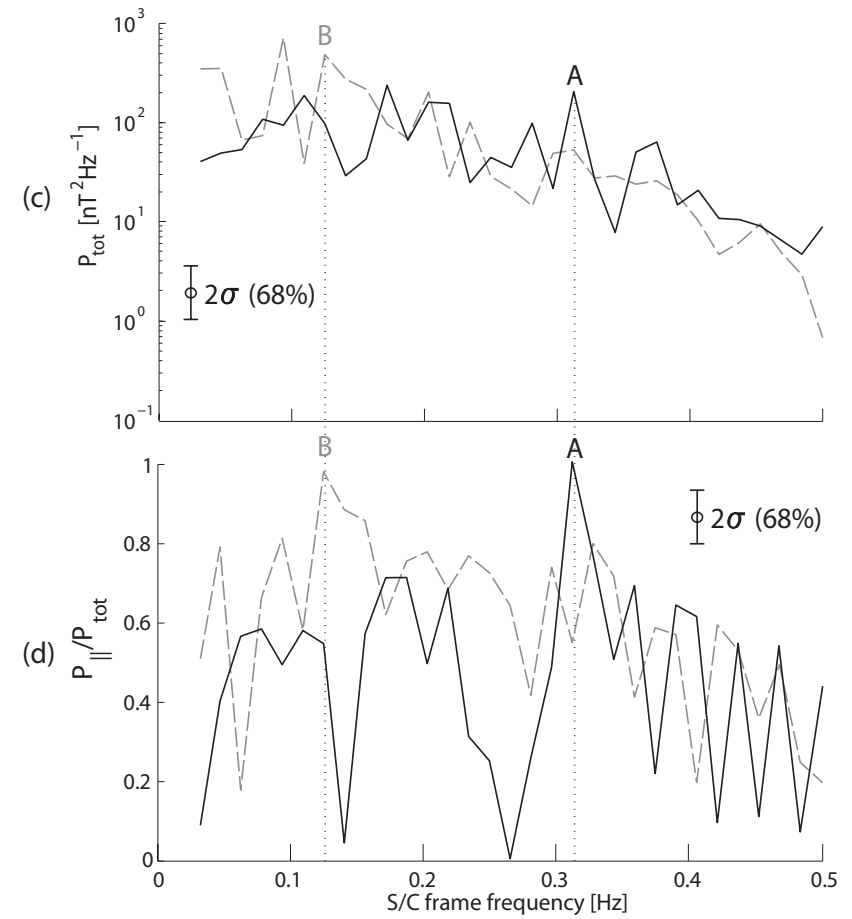

Figure 2. (c) Total power spectral density and (d) ratio between compressional and total poser spectral density for the two time intervals (A and B) marked in Fig. 2a and b, based on Venus Express data.

between the bow shock and the locations where MM structures were observed are $L_{\mathrm{A}} \approx 2710 \mathrm{~km}$ and $L_{\mathrm{B}} \approx 4050 \mathrm{~km}$. Therewith, the HT-model (Eq. 1) yields estimates of the MM sizes of $14 \pm 3 \rho_{\mathrm{p}}$ in A and of $24 \pm 5 \rho_{\mathrm{p}}$ in $\mathrm{B}$, where we assumed a relative error of $10 \%$ in the measured distance $L$.

Since the measured and calculated MM sizes remain within the error tolerances, we interpret that the MM structures originated at the bow shock.

\subsection{P/Halley magnetosheath}

In contrast to the Venus Express case above, Giotto entered the magnetosheath of comet $\mathrm{P} / \mathrm{Halley}$ on the dusk side at about $(-0.32,1.03,-0.14) \times 10^{6} \mathrm{~km}$ in HSE coordinates, at 19:37 UT. According to the identification criteria stated above, and the assumption that these criteria are fulfilled for a continuous period of more than $120 \mathrm{~s}$ with maximum $30 \mathrm{~s}$ interruption, we find three time intervals with strong compressive fluctuations due to MM structures between 21:00-23:30 UT on 13 March 1986 and 01:3003:00 UT on 14 March 1986, when Giotto was clearly in the magnetosheath of $\mathrm{P} /$ Halley. We exclude the time interval for which Giotto was in the magnetic pileup boundary region, as similar magnetic structures have shown to be compressional fast-mode magnetosonic waves (see Mazelle et al., 1991). Figure 3a, b, and c display the magnetic field and electron density data from Giotto on 13 March 1986 between 23:0523:08 and 23:12-23:30 UT. The gray shaded areas C, D and E mark the three found MM structures. Although the fourth time interval (gray shaded area F) does not fulfill our criteria perfectly, it has been identified as a MM wave in earlier studies (Glassmeier et al., 1993). In the following we examine these four MM structures.

Spectral analysis performed on these intervals yields clear peaks at $0.046,0.039$ and $0.023 \mathrm{~Hz}$ (vertical lines in Fig. 3a, $\mathrm{b}$, and c), which correspond to periods of 22, 26 and $43 \mathrm{~s}$, for $\mathrm{E}, \mathrm{D}$ and $\mathrm{C} / \mathrm{F}$ respectively. The median of the alpha particle velocity during the MM interval is $V_{\mathrm{C}}=(-81,22,2) \mathrm{km} \mathrm{s}^{-1}$ for $\mathrm{C}, V_{\mathrm{D}}=(-67,5,11) \mathrm{km} \mathrm{s}^{-1}, V_{\mathrm{E}}=(-77,12,17) \mathrm{km} \mathrm{s}^{-1}$, and $V_{\mathrm{F}}=(-54,22,0) \mathrm{km} \mathrm{s}^{-1}$ for $\mathrm{D}, \mathrm{E}$ and $\mathrm{F}$ respectively. The water group gyroradius is obtained from the median magnetic field and the pickup velocity, which is assumed to be the bulk flow velocity of the alpha particles (see above), and is approximately $2000 \pm 1000 \mathrm{~km}$ for C, $1600 \pm 800 \mathrm{~km}$, $1500 \pm 800 \mathrm{~km}$ and $1000 \pm 500 \mathrm{~km}$ for D, E and F respectively. Since the IMS plasma instrument has detector fieldof-view complications (see above), we assume a relative error of $50 \%$ for the measured plasma velocity. This yields an 


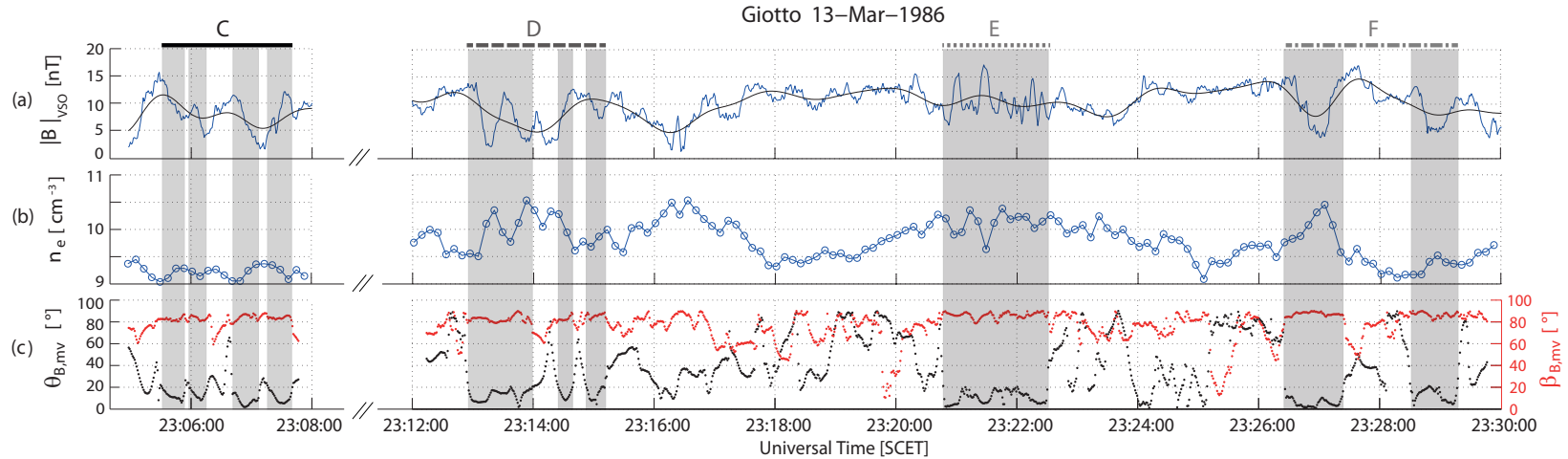

Figure 3. Time series of (a) total magnetic field, (b) electron density and (c) angles $(\theta$ and $\beta$ ) between the maximum and minimum variance directions and the background magnetic field, based on Giotto data.

(d)

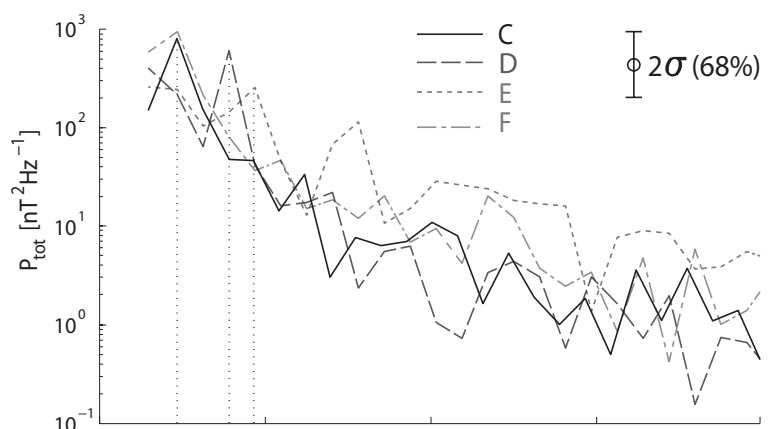

(e)

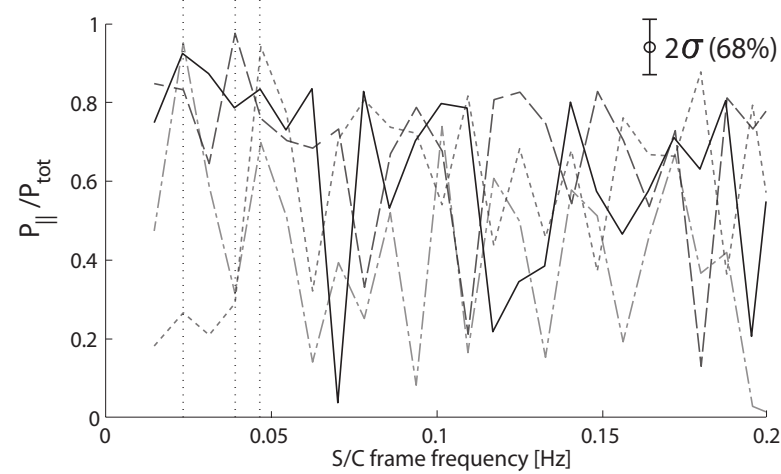

Figure 3. (d) Total power spectral density and (e) ratio between compressional and total poser spectral density for the four time intervals (C, D, E and F) marked in Fig. 3a, b, and c, based on Giotto data. Only the relevant frequency range is shown.

observed MM size of about $1 \pm 1 \rho_{\mathrm{H}_{2} \mathrm{O}}$ for $\mathrm{D}$ and $\mathrm{E}$, and about $2 \pm 1 \rho_{\mathrm{H}_{2} \mathrm{O}}$ for $\mathrm{C}$ and $\mathrm{F}$. Table 1 summarizes the MM time intervals, periods, gyroradii and scale sizes.

The results show that the observed MM structures are most likely the result from the local pickup process. Tsurutani et al. (1999) argued that pickup ions at comet GiacobiniZinner just outside the magnetic pileup region may cause MM structures. Indeed, Giotto enters the pileup region at about $23: 38 \mathrm{UT}$ at $(-0.30,0.97,-0.13) \times 10^{5} \mathrm{~km}$. Therefore, we conclude that cometary mirror mode fluctuations are created locally in the middle of the magnetosheath and are not associated with generation at the bow shock and subsequent convection and diffusion.

\section{Summary and discussion}

Our study suggests that at Venus the dominant mirror mode source is most likely the bow shock, while in the cometary magnetosheath the source is more likely associated with a local ion pickup process. This statement, however, is based on case studies and needs to be confirmed by statistical analyses. The relevance of the pickup process at Venus should also be evaluated. The pickup process is dependent on solar ultra-violet flux (e.g., Wilken et al., 1987), which varies strongly with solar cycle. Hence, magnetosheath spectra and scale sizes of MM structures could also be solar cycle dependent.

The notion of mirror mode diffusion, though rather simple, seems to be valid not only in the magnetosheath of magnetized planets and in the heliosheath but also in the magnetosheath of induced magnetospheres. Lucek et al. (1999a) and Volwerk et al. (2008) also found that the peak in the power spectrum shifts to lower frequencies as the spacecraft moves deeper into the magnetosheaths at Earth and Venus respectively. Diffusion can explain why the observed MM sizes are larger deeper in the magnetosheath. Thus, we find that the diffusion model constitutes a useful analysis tool for studying complex plasma processes. The model yields a reasonable estimate of the distance of identified MM structures to their source region (if the ambient plasma conditions are known), which is also a measure of the relative importance of different MM sources.

We have confirmed the applicability of the Lucek et al. (1999a, b) method for P/Halley using electron data. It should be noted that the detected MM structures have a scale size comparable to the water group gyroradius. Tsurutani et al. (1999), however, showed that at Comet Giacobini-Zinner the size of the MM structure is $\sim 12$ times 
the (local) heavy ion $\left(\mathrm{H}_{2} \mathrm{O}^{+}\right)$gyroradius. Interestingly, the production rate of $\mathrm{P} / \mathrm{Halley}$ compared to Giacobini-Zinner is $\sim 10$ times higher. It might be that the higher density of neutrals at P/Halley inhibited the growth of the MM structures, whereas at Giacobini-Zinner it is possible that the MM structures, similar to the case of Venus, evolve in time while they convect away from their source region. Again, a possible mechanism could be a turbulent diffusion process of the MM. This should be evaluated by adapting the HT-model for the heavy $\mathrm{H}_{2} \mathrm{O}^{+}$ions.

It is worth while to mention that the ion pickup process is also important for the heliosheath mirror modes. The termination shock is probably stronger than that of comet $\mathrm{P} / \mathrm{Halley}$, so shock heating could play an even more essential role there. Fortunately, Voyager spacecraft is measuring magnetic field in situ in the heliosheath. It would be interesting to compare the mirror mode size with the pickup ion scales of proton, helium, or other abundant species coming from the local interstellar medium.

Acknowledgements. We thank F. M. Neubauer and co-workers for providing Giotto magnetic field data and M. Bentley for evaluating the Giotto position data. We acknowledge AMDA and K. H. Glassmeier for providing the plasma data of VeX and Giotto. The work by D. Schmid was partially supported by FWF under grant P25257-N27. Part of the work was supported by FP7 under grant 313038/STORM.

Topical Editor L. Blomberg thanks O. A. Pokhotelov and one anonymous referee for their help in evaluating this paper.

\section{References}

Balsiger, H., Altwegg, K., Bühler, F., Geiss, J., Ghielmetti, A. G., Goldstein, B. E., Goldstein, R., Huntress, W. T., Ip, W.-H., Lazarus, A. J., Meier, A., Neugebauer, M., Rettenmund, U., Rosenbauer, H., Schwenn, R., Sharp, R. D., Shelley, E. G., Ungstrup, E., and Young, D. T.: Ion composition and dynamics at comet Halley, Nature, 321, 330-334, doi:10.1038/321330a0, 1986.

Barabash, S., Sauvaud, J.-A., Gunell, H., Andersson, H., Brinkfeldt, A. G. K., Holmström, M., Lundin, R., Yamauchi, M., Asamura, K., Baumjohann, W., Zhang, T., Coates, A., Linder, D., Kataria, D., Curtis, C., Hsieh, K., Sandel, B., Fedorov, A., Mazelle, C., Thocaven, J.-J., Grande, M., Koskinen, H., Kallio, E., Säles, T., Riihela, P., Kozyra, J., Krupp, N., Woch, J., Luhmann, J., McKenna-Lawlor, S., Orsini, S., Cerulli-Irelli, R., Mura, M., Milillo, M., Maggi, M., Roelof, E., Brandt, P., Russell, C., Szego, K., Winningham, J., Frahm, R., Scherrer, J., Sharber, J., Wurz, P., and Bochsler, P.: The Analyser of Space Plasmas and Energetic Atoms (ASPERA-4) for the Venus Express mission, Planet. Space Sci., 55, 1772-1792, 2007.

Baumjohann, W., Treumann, R. A., Georgescu, E., Haerendel, G., Fornacon, K.-H., and Auster, U.: Waveform and packet structure of lion roars, Ann. Geophys., 17, 1528-1534, doi:10.1007/s00585-999-1528-9, 1999.
Bavassano-Cattaneo, M. B., Basile, C., Moreno, G., and Richardson, J. D.: Evolution of mirror structures in the magnetosheath of Saturn from the bow shock to the magnetopause, J. Geophys. Res., 103, 11961-11972, 1998.

Czaykowska, A., Bauer, T. M., Treumann, R. A., and Baumjohann, W.: Mirror waves downstream of the quasi-perpendicular bow shock, J. Geophys. Res., 103, 4747-4753, 1998.

Delva, M., Bertucci, C., Schwingenschuh, K., Volwerk, M., and Romanelli, N.: Magnetic pileup boundary and field draping at Comet Halley, Planet. Space Sci., online first, doi:10.1016/j.pss.2014.02.010, 2014.

Erdõs, G. and Balogh, A.: Statistical properties of mirror mode structures observed by Ulysses in the magnetosheath of Jupiter, J. Geophys. Res., 101, 1-12, 1993.

Eriksson, A. I.: Spectral analysis, in: Analysis Methods for MultiSpacecraft Data, edited by: Paschmann, G. and Daly, P., 307322, ESA, Noordwijk, 1998.

Gary, S., Fuselier, S., and Anderson, B. J.: Ion anisotropy instabilities in the magnetosheath, J. Geophys. Res., 98, 1481-1488, 1993.

Glassmeier, K. H., Motschmann, U., Mazalle, C., Neubauer, F. M., Sauer, K., Fuselier, S. A., and Acuna, M. H.: Mirror modes and fast magnetoaucoustic waves near the magnetic pileup boundary of comet P/Halley, J. Geophys. Res., 98, 20955-20964, 1993.

Hasegawa, A.: Drift mirror instability in the magnetosphere, Phys. Fluids, 12, 2642-2650, 1969.

Hasegawa, A. and Tsurutani, B. T.: Mirror mode expansion in planetary magnetosheaths: Bohm-like diffusion, Phys. Rev. Lett., 107, 245005, doi:10.1103/PhysRevLett.107.245005, 2011.

Johnstone, A. D., Coates, A. J., Wilken, B., Studemann, W., Weiss, W., Irelli, R. C., Formisano, V., Borg, H., Olsen, S., Winningham, J. D., Bryant, D. A., and Kellock, S. J.: The Giotto three-dimensional positive ion analyser, J. Phys. E, 20, 795, doi:10.1088/0022-3735, 1987.

Lucek, E. A., Dunlop, M. W., Balogh, A., Cargill, P., Baumjohann, W., Georgescu, E., Haerendel, G., and Fornaçon, K.-H.: Mirror mode structures observed in the dawn-side magnetosheath by Equator-S, Geophys. Res. Lett., 26, 2159-2162, 1999a.

Lucek, E. A., Dunlop, M. W., Balogh, A., Cargill, P., Baumjohann, W., Georgescu, E., Haerendel, G., and Fornacon, G.-H.: Identification of magnetosheath mirror modes in Equator-S magnetic field data, Ann. Geophys., 17, 1560-1573, doi:10.1007/s00585999-1560-9, 1999b.

Martinecz, C.: The Venus plasma environment: a comparison of Venus Express ASPERA-4 measurements with 3D hybrid simulations, dissertation, TU Braunschweig, available at: http://dnb. ddb.de (last access: 21 March 2014), 2008.

Mazelle, C., Belmont, G., Glassmeier, K.-H., Quéau, D. L., and Réme, H.: Ultra low frequency waves at the magnetic pile-up boundary of comet P/Halley, Adv. Space Res., 11, 73-77, 1991.

Narita, Y., Glassmeier, K.-H., Fornaçon, K.-H., Richter, I., Schäfer, S., Motschmann, U., Dandouras, I., Rème, H., and Georgescu, E.: Low-frequency wave characteristics in the upstream and downstream regime of the terrestrial bow shock, J. Geophys. Res., 111, A01203, doi:10.1029/2005JA011231, 2006.

Neubauer, F. M., Glassmeier, K. H., Pohl, M., Raeder, J., Acuna, M. H., Burlaga, L. F., Ness, N. F., Musmann, G., Mariani, F., Wallis, M. K., Ungstrup, E., and Schmidt, H. U.: First results 
from the Giotto magnetometer experiment at comet Halley, Nature, 321, 352-355, doi:10.1038/321352a0, 1986.

Price, C. P., Swift, D. W., and Lee, L.-C.: Numerical simulation of nonoscillatory mirror waves at the Earth's magnetosheath, J. Geophys. Res., 91, 101-112, doi:10.1029/JA091iA01p00101, 1986.

Remya, B., Reddy, R. V., Tsurutani, B. T., Lakhina, G. S., and Echer, E.: Ion temperature anisotropy instabilities in planetary magnetosheaths, J. Geophys. Res., 118, 785-793, doi:10.1002/jgra.50091, 2013.

Sonnerup, B. U. Ö. and Scheible, M.: Minimum and maximum variance analysis, in: Analysis Methods for Multi-Spacecraft Data, edited by: Paschmann, G. and Daly, P., 185-220, ESA, Noordwijk, 1998.

Tsurutani, B. T., Smith, E. J., Anderson, R. R., Ogilvie, K. W., Scudder, J. D., Baker, D. N., and Bame, S. J.: Lion roars and nonoscillatory drift mode mirror waves in the magnetosheath, J. Geophys. Res., 87, 6060-6072, 1982.

Tsurutani, B. T., Lakhina, G. S., Smith, E. J., Buti, B., Moses, S. L., Coroniti, F. V., Brinca, A. L., Slavin, J. A., and Zwickl, R. D.: Mirror mode structures and ELF plasma waves in the GiacobiniZinner magnetosheath, Nonlinear Proc. Geoph., 6, 229-234, 1999.

Violante, L., Bavassano-Cattaneo, M. B., Moreno, G., and Richardson, J. D.: Observations of mirror waves and plasma depletion layer upstream of Saturn's magnetopause, J. Geophys. Res., 100, 12047-12055, doi:10.1029/94JA02703, 1995.

Volwerk, M., Zhang, T. L., Delva, M., Vörös, Z., and Baumjohann, W.: First identification of mirror mode waves in Venus' magnetosheath?, Geophys. Res. Lett., 35, L12204, doi:10.1029/2008GL033621, 2008.
Wilken, B., Johnstone, A., Coates, A., Amata, E., and Borg, H.: Pick-up ions at Comet P/Halley's bow shock - Observations with the IIS spectrometer on Giotto, Astron. Astrophys., 187, 153159, 1987.

Zhang, T., Baumjohann, W., Delva, M., Auster, H.-U., Balogh, A., Russell, C., Barabash, S., Balikhin, M., Berghofer, G., Biernat, H., Lammer, H., Lichtenegger, H., Magnes, W., Nakamura, R., Penz, T., Schwingenschuh, K., Vörös, Z., Zambelli, W., Fornaçon, K.-H., Glassmeier, K.-H., Richter, I., Carr, C., Kudela, K., Shi, J., Zhao, H., Motschmann, U., and Lebreton, J.-P.: Magnetic field investigation of the Venus plasma environment: Expected new results from Venus Express, Planet. Space Sci., 54, 13361343, 2006.

Zhang, T. L., Delva, M., Baumjohann, W., Auster, H.-U., Carr, C., Russell, C. T., Barabash, S., Balikhin, M., Kudela, K., Berghofer, G., Biernat, H. K., Lammer, H., Lichtenegger, H., Magnes, W., Nakamura, R., Schwingenschuh, K., Volwerk, M., Vörös, Z., Zambelli, W., Fornaçon, K.-H., Glassmeier, K.-H., Richter, I., Balogh, A., Schwarzl, H., Pope, S. A., Shi, J. K., Wang, C., Motschmann, U., and Lebreton, J.-P.: Little or no solar wind enters Venus' atmosphere at solar minimum, Nature, 450, 654-656, 2007.

Zhang, T. L., Baumjohann, W., Russell, C. T., Jian, L. K., Wang, C., Cao, J. B., Balikhin, M., Blanco-Cano, X., Delva, M., and Volwerk, M.: Mirror mode structures in the solar wind at $0.72 \mathrm{AU}, \mathrm{J}$. Geophys. Res., 114, A10107, doi:10.1029/2009JA014103, 2009. 